\title{
Risk Factors for Microvascular Complications of Diabetes in a High-Risk Middle East Population
}

\author{
Sohaila Cheema (D), ${ }^{1}$ Patrick Maisonneuve, ${ }^{2}$ Mahmoud Zirie, ${ }^{3}$ Amin Jayyousi, ${ }^{3}$ \\ Hekmat Alrouh, ${ }^{1}$ Amit Abraham, ${ }^{1}$ Sura Al-Samraye, ${ }^{1}$ Ziyad Mahfoud, ${ }^{1}$ \\ Ibrahim Mohammed Al-Janahi, ${ }^{3}$ Buthaina Ibrahim, ${ }^{3}$ Albert B. Lowenfels, ${ }^{4}$ \\ and Ravinder Mamtani ${ }^{1}$ \\ ${ }^{1}$ Institute for Population Health, Weill Cornell Medicine-Qatar, Education City, Qatar Foundation, P.O. Box 24144, Doha, Qatar \\ ${ }^{2}$ Division of Epidemiology and Biostatistics, European Institute of Oncology, Milan, Italy \\ ${ }^{3}$ Department of Internal Medicine, Hamad Medical Corporation, Doha, Qatar \\ ${ }^{4}$ Department of Surgery and Department of Family Medicine, New York Medical College, Valhalla, NY, USA
}

Correspondence should be addressed to Sohaila Cheema; soc2005@qatar-med.cornell.edu

Received 23 October 2017; Revised 18 March 2018; Accepted 3 April 2018; Published 2 July 2018

Academic Editor: Daniela Foti

Copyright ( 2018 Sohaila Cheema et al. This is an open access article distributed under the Creative Commons Attribution License, which permits unrestricted use, distribution, and reproduction in any medium, provided the original work is properly cited.

\begin{abstract}
Aims. Much of the diabetes burden is caused by its complications. This cross-sectional study aimed to determine the prevalence and risk factors for diabetic microvascular complications (retinopathy, nephropathy, and neuropathy) in a high-risk population. Methods. We collected information via a structured questionnaire and directly from the patient's record on 1034 adult type 2 diabetic patients who were attending outpatient clinics in Qatar. Results. The mean age of the patients was $55 \pm 10$ years, and the mean duration of diabetes was $12.4 \pm 8.9$ years. Forty-five percent had one or more microvascular complications. Shared risk factors for multiplicity and for individual complications included family history, severity and duration of diabetes, and hypertension, but some risk factors were specific for individual microvascular complications. Early age at onset of diabetes was strongly associated with multiplicity of complications $(P=0.0003)$. Conclusions. About half the diabetics in this high-risk population had one or more microvascular complications. Several well-established risk factors were associated with multiplicity and individual microvascular complications, but each separate microvascular complication was linked to a somewhat different constellation of risk factors.
\end{abstract}

\section{Introduction}

Bahrain, Kuwait, Oman, Qatar, Saudi Arabia, and the United Arab Emirates constitute the members of the Gulf Cooperation Council (GCC) and have high prevalence rates of type 2 diabetes [1]. In Qatar, the burden of diabetes is particularly high, with an estimated 4.35 years of disability adjusted life years directly attributable to this cause [2].

Elsewhere in the Middle East, diabetes is also prevalent, mirroring the current global epidemic of diabetes [3]. The high prevalence of obesity and lack of exercise, along with the possibility of region-specific genetic mutations associated with diabetes, may be the explanation, thereby further confirming the genetic peculiarity of some populations $[4,5]$.
Complications of diabetes contribute greatly to the increased mortality and morbidity associated with this disease. Diabetic complications are customarily divided into two main categories: macrovascular complications, including heart disease and stroke, and microvascular complications, which include retinopathy, nephropathy, and neuropathy. In the multinational A1chieve study, a large global study, based on nearly 68,000 type 2 diabetics, microvascular complications accounted for about half the total number of complications [6].

Microvascular complications, the focus of this report, are a leading cause of blindness, kidney failure, and lower limb amputation. It is unclear why some diabetic patients develop microvascular complications and other patients do not. 
Previous investigators have pointed out that the links between the various microvascular complications need further exploration $[7,8]$ and only limited data are available about their prevalence in Qatar.

The aims of this study performed in the type 2 diabetic adult population of Qatar were to (1) examine the frequency and overlap of microvascular complications, (2) determine risk factors for individual diabetic complications, and (3) determine if smoking, a risk factor for diabetes, was also associated with microvascular complications.

\section{Materials and Methods}

2.1. Study Subjects. The information in this report came from a cross-sectional survey conducted among patients attending the outpatient clinics of Hamad General Hospital, the largest tertiary care hospital in Doha, the capital of Qatar. Data were collected from June 2013 to January 2015 using a detailed questionnaire (supplementary file available on request) covering patient background information, health status, tobacco use, chronic disease history, and family history. Trained personnel from the Division of Global and Public Health (now Institute for Population Health), Weill Cornell Medicine-Qatar, completed the questionnaire while interviewing patients at the time of their clinic visit. The patients were recruited from the diabetic, endocrine, gastroenterology, ophthalmology, nephrology, dietetic, podiatry, and smoking cessation clinics. The inclusion criteria for recruitment were age $\geq 18$ and previous diagnosis of diabetes. Exclusion criteria included subjects less than 18 years of age, subjects diagnosed with type I diabetes, and those with gestational diabetes. In addition to information obtained directly from patients, the interviewer also collected information from the patient's chart relating to current findings on physical examination, medication use, and laboratory data. Detailed smoking questions were included in the questionnaire because smoking is a known risk factor for diabetes, but its relationship to various complications has not been well documented. Because of the low prevalence of smoking in the female population of this region (less than one percent of Qatari women smoke, and the prevalence of smoking in non-Qatari women is only about 3\%) [9], we prematurely stopped the enrollment of female diabetic patients in the study on May 15, 2014, concentrating our resources and effort to male diabetics. To achieve diagnostic homogeneity in the study, we limited participants to adults (age 18 or more) diagnosed with type 2 diabetes.

2.2. Ethical Considerations. The study was reviewed and approved by the Research Office at Weill Cornell MedicineQatar and the Research Committee at Hamad Medical Corporation. Patient participation in the study was entirely voluntary, and less than $2 \%$ of patients who were approached opted out of the study. Prior to the interview, a statement regarding the study embodying the elements of consent was read out to the subjects and verbal consent was obtained from each recruited subject.
2.3. Statistical Analysis. The study sample size ( 800 men) was calculated to detect a $10 \%$ difference in the prevalence of complications among male diabetic smokers, corresponding to an odds ratio $=1.5$, assuming that $45 \%$ of men ever smoked and that the prevalence of complications is $50 \%$ in nonsmokers, using a two-sided $Z$ test with pooled variance. Association between sociodemographic, lifestyle, and past medical history characteristics and the number of complications was assessed with the Mantel-Haenszel test for trend (categorical variables) and analysis of variance (ANOVA) for continuous variables. Odds ratios (OR) and 95\% confidence intervals (CI) for the association between patients' characteristics and microvascular disease were estimated using logistic regression models adjusted for age, gender, and duration of diabetes. Multivariable logistic regression was then used to assess independence of the associations, fitting together all the variables that were associated with outcome in the previous analysis. Factors considered for inclusion in the multivariable analysis included the following: age (per 10-year increase), gender, duration of diabetes (10 or more years versus less than 10 years), education level, first degree family history of diabetes (yes versus no), insulin treatment (yes versus no), body mass index (per $5 \mathrm{~kg} / \mathrm{m}^{2}$ ), history of hypertension under treatment, blood pressure (stage 1 or 2 hypertension $(\geq 140 / \geq 90)$ versus normal or prehypertension), LDL level (per 1 unit increase), HbA1c blood level (per 1 unit increase), and high creatinine level (>106 versus $\leq 106 \mu \mathrm{mol} / \mathrm{L})$. All analyses were performed with SAS version 9.4 (Cary, NC). All $P$ values were twosided. $P$ values $<0.05$ were considered statistically significant.

\section{Results and Discussion}

3.1. Results. Table 1 contains information about the 1034 patients with type 2 diabetes mellitus who were included in the analysis. The mean age ( \pm standard deviation) of patients was 55 years \pm 10 years (range 18-84). The average patient age at onset of diabetes was $43 \pm 11$ years, the average duration of diabetes was $12.4 \pm 9.0$ years, and the mean glycated hemoglobin (HbAlc) level was $8.3 \pm 1.8 \%$. Forty-six percent of male patients were current or former smokers, while only $2(0.8 \%)$ of female patients ever smoked. Gender-specific participants' characteristics are available in Supplementary Tables $1 \mathrm{a}$ and $1 \mathrm{~b}$.

When compared to patients with no complications, several variables were associated with multiplicity of complications. These included age, gender, education, family history, duration, and age at onset of diabetes, HbAlc level, diabetic therapy, LDL, creatinine levels, and elevated systolic blood pressure.

Table 2 and Figure 1 provide information about the frequency of individual and combinations of complications, overall and by gender. We stratified microvascular complications into the same major groups used by other authors: nephropathy, neuropathy, and retinopathy [4]. Microvascular complications were present at the time of the survey in $48.4 \%(n=500)$ of patients; $30.8 \%(n=318)$ had one complication; $13.5 \%(n=140)$ had two microvascular complications, and only $4.1 \%(n=42)$ had all three 
TABLE 1: Participant characteristics.

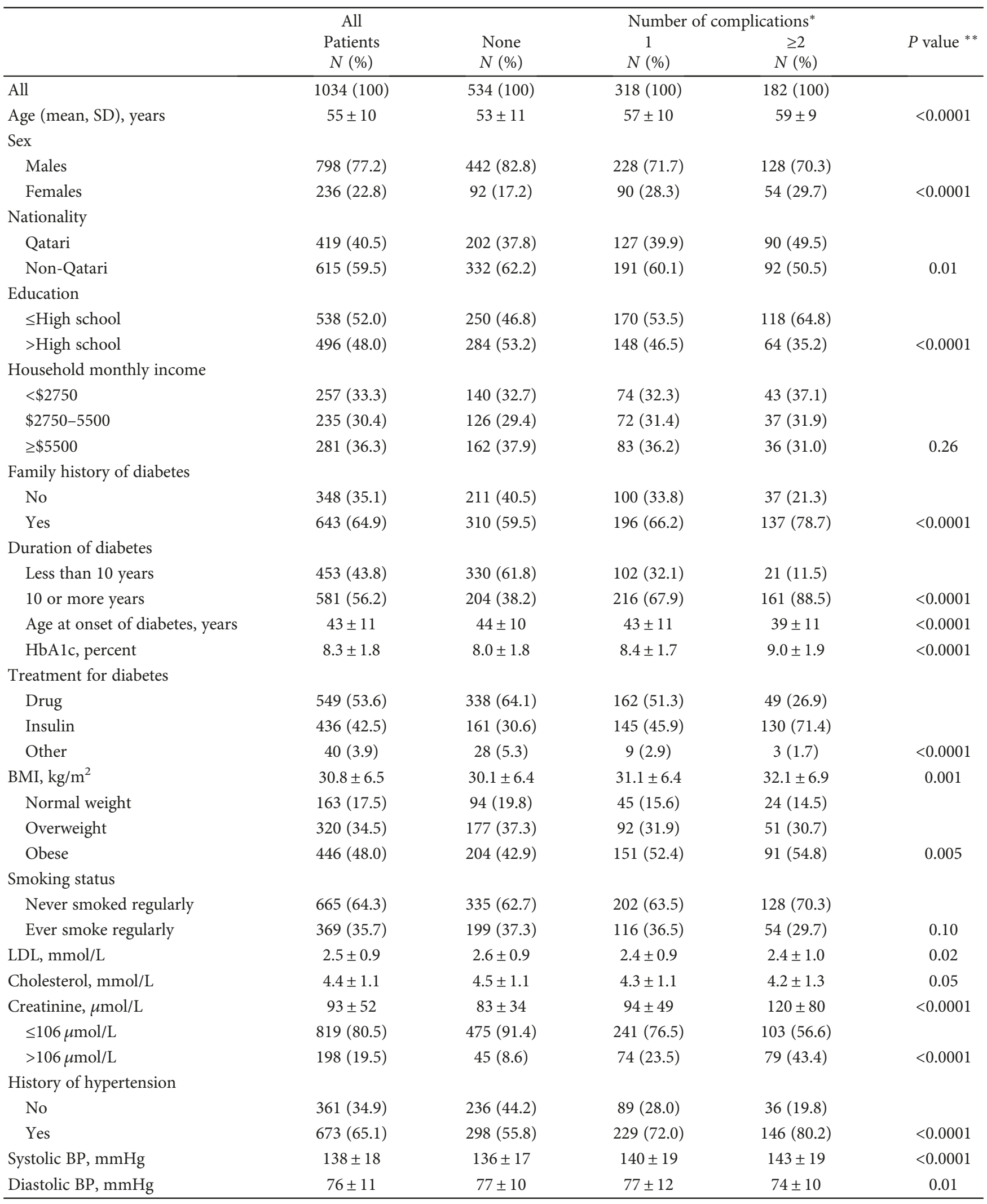

${ }^{*}$ Retinopathy, nephropathy, and neuropathy following the diagnosis of diabetes. ${ }^{*} P$ value using the Mantel-Haenszel test for trend for categorical variables and ANOVA for continuous variables. SD: standard deviation; HbA1c: hemoglobin A1c; LDL: low-density lipoprotein; BP: blood pressure. 
TABLe 2: Prevalence of major microvascular complications in 1034 patients with type 2 diabetes in Qatar, by gender.

\begin{tabular}{|c|c|c|c|c|}
\hline & $\begin{array}{c}\text { All } \\
N(\%)\end{array}$ & $\begin{array}{l}\text { Males } \\
N(\%) \\
\end{array}$ & $\begin{array}{c}\text { Females } \\
N(\%)\end{array}$ & $P$ value \\
\hline Total number of patients & $1034(100)$ & $798(100)$ & $236(100)$ & \\
\hline Number of complications & & & & $<0.0001$ \\
\hline None & $534(51.6)$ & $442(55.4)$ & $92(39.0)$ & \\
\hline One complication & $318(30.8)$ & $228(28.6)$ & $90(38.1)$ & \\
\hline Retinopathy & $108(10.4)$ & $81(10.2)$ & $27(11.4)$ & 0.55 \\
\hline Nephropathy & $55(5.3)$ & $50(6.3)$ & $5(2.1)$ & 0.01 \\
\hline Neuropathy & $155(15.0)$ & $97(12.2)$ & $58(24.6)$ & $<0.0001$ \\
\hline Two complications & $140(13.5)$ & $97(12.2)$ & $43(18.2)$ & \\
\hline Retinopathy + nephropathy & $37(3.6)$ & $33(4.1)$ & $4(1.7)$ & 0.11 \\
\hline Retinopathy + neuropathy & $79(7.6)$ & $45(5.6)$ & $34(14.4)$ & $<0.0001$ \\
\hline Nephropathy + neuropathy & $24(2.3)$ & $19(2.4)$ & $5(2.1)$ & 1.00 \\
\hline Three complications & $42(4.1)$ & $31(3.9)$ & $11(4.7)$ & 0.58 \\
\hline \multicolumn{5}{|l|}{ Patterns of combined complications } \\
\hline Retinopathy ( \pm other complications) & $266(25.7)$ & $190(23.8)$ & $76(32.2)$ & 0.01 \\
\hline Nephropathy ( \pm other complications) & $158(15.3)$ & $133(16.7)$ & $25(10.6)$ & 0.02 \\
\hline Neuropathy ( \pm other complications) & $300(29.0)$ & $192(24.1)$ & $108(45.8)$ & $<0.0001$ \\
\hline
\end{tabular}

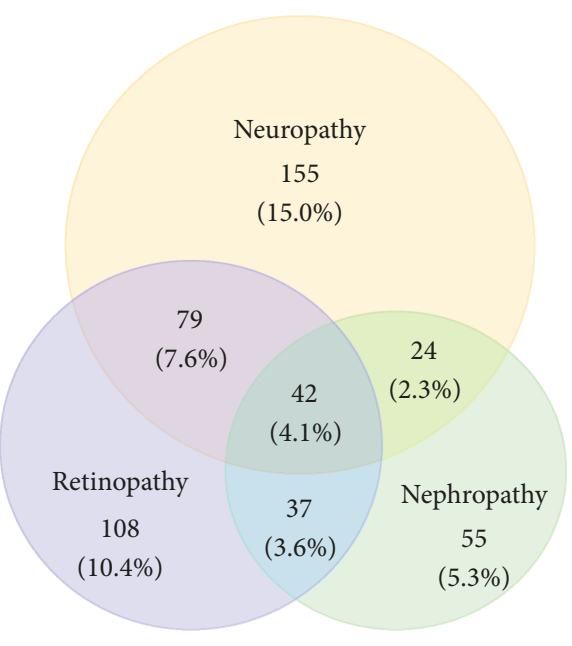

NONE: $534(51.6 \%)$

FIGURE 1: Prevalence of major microvascular complications in 1034 patients with type 2 diabetes in Qatar.

microvascular complications. Significantly more women than men reported any microvascular complication $(61.0 \%$ versus $44.6 \%, P<0.0001)$.

Three hundred $(29.0 \%)$ patients reported neuropathy, 266 (25.7\%) retinopathy, and 158 (15.3\%) nephropathy. Female patients had higher prevalence of neuropathy (45.8\% versus $24.1 \%, P<0.0001)$ and retinopathy $(32.2 \%$ versus $23.8 \%, P=0.01)$ but lower prevalence of nephropathy (10.6\% versus $16.7 \%, P=0.02$ ) than male patients.

The mean age at development of all three microvascular complications was similar and ranged from $53.3 \pm 9.2$ years (neuropathy) to $55.2 \pm 10.6$ years (nephropathy). Similarly, the mean interval between the diagnosis of diabetes and the development of microvascular complications was also similar, ranging from $12.9 \pm 8.5$ years (neuropathy) to $14.4 \pm 8.1$ years (retinopathy).

Table 3 presents the results of a multivariable analysis of all factors that were associated with diabetes in after sole adjustment for age, gender, and duration of diabetes (results available in Supplementary Table 2). Several variables were significantly associated with one or more microvascular complications, but HbA1c was the only variable significantly associated with all three complications in the fully adjusted model. Each of the three major microvascular complications had a distinct pattern of associated risk factors.

3.2. Discussion. This study focused on the prevalence of major complications in previously diagnosed adult male diabetics attending outpatient clinics at Hamad General Hospital that is a major provider of health care for the diabetic population of Doha, the capital of Qatar. In these adult type 2 diabetic patients, about half developed one or more microvascular complications 12-14 years after the onset of diabetes. In patients who did develop complications, about two-thirds developed a single complication and one-third developed two or more complications.

The role of smoking as an important risk factor for diabetes needs further emphasis: about half the subjects in this group of male diabetics were current or ex-smokers. The increased prevalence of smoking in these patients is understandable since smoking is known to be an important risk factor for diabetes [10]. However, even though smoking is known to be related with diabetes, smoking status (ever versus never) was unrelated to the presence or absence of microvascular complications nor to the development of any single microvascular complication, perhaps because smoking has equal effects on the microcirculation in the various sites were microvascular complications are diagnosed. 
TABLE 3: Multivariable analysis of factors associated with microvascular complications.

\begin{tabular}{|c|c|c|c|c|c|c|}
\hline \multirow{2}{*}{ Variable } & \multicolumn{2}{|c|}{ Retinopathy } & \multicolumn{2}{|c|}{ Nephropathy } & \multicolumn{2}{|c|}{ Neuropathy* } \\
\hline & OR $(95 \% \mathrm{CI})$ & $P$ value & OR $(95 \% \mathrm{CI})$ & $P$ value & OR $(95 \% \mathrm{CI})$ & $P$ value \\
\hline Age (per 10 years) & $1.19(1.00-1.43)$ & 0.05 & $0.87(0.69-1.11)$ & 0.27 & $1.18(1.00-1.39)$ & 0.06 \\
\hline Gender (female versus male) & $1.34(0.92-1.95)$ & 0.13 & $0.95(0.53-1.70)$ & 0.85 & $2.07(1.44-2.97)$ & $<0.0001$ \\
\hline Duration of $\mathrm{DM} \geq 10$ years & $3.83(2.56-5.72)$ & $<0.0001$ & $1.48(0.87-2.49)$ & 0.15 & $2.77(1.92-3.99)$ & $<0.0001$ \\
\hline Education (>high school) & $0.79(0.57-1.10)$ & 0.16 & $0.61(0.38-0.96)$ & 0.03 & - & \\
\hline Family history of DM (yes versus no) & - & & - & & $1.63(1.15-2.30)$ & 0.006 \\
\hline HbA1c level (per \% increase) & $1.14(1.04-1.25)$ & 0.006 & $1.15(1.00-1.33)$ & 0.05 & $1.12(1.02-1.23)$ & 0.01 \\
\hline DM treatment (insulin versus drug) & $1.61(1.14-2.26)$ & 0.006 & $2.05(1.25-3.39)$ & 0.005 & $1.26(0.90-1.75)$ & 0.18 \\
\hline Body mass index (per $5 \mathrm{~kg} / \mathrm{m}^{2}$ ) & - & & - & & $1.29(1.14-1.47)$ & $<0.0001$ \\
\hline LDL (per mmol/L increase) & - & & $0.82(0.64-1.05)$ & 0.11 & - & \\
\hline Creatinine (>106 versus $\leq 106 \mu \mathrm{mol} / \mathrm{L}$ ) & $1.63(1.11-2.41)$ & 0.01 & $23.9(14.7-38.8)$ & $<0.0001$ & $1.36(0.93-1.99)$ & 0.12 \\
\hline Hypertension or high BP & $1.87(1.29-2.71)^{1}$ & 0.001 & $2.03(1.15-3.59)^{1}$ & 0.01 & $1.31(0.97-1.78)^{2}$ & 0.08 \\
\hline
\end{tabular}

Odds ratios (OR) and 95\% confidence intervals (CI) obtained from multivariable logistic regression model with all displayed variables fitted simultaneously.

${ }^{*}$ Foot ulcer and amputation. ${ }^{1}$ History of hypertension, undergoing treatment. ${ }^{2}$ Systolic blood pressure $\geq 140$ or diastolic blood pressure $\geq 90$ at time of survey

In general, factors that were associated with multiplicity of microvascular complications listed in Table 1 were similar to those related to each individual complication. Age at onset of diabetes was strongly associated with increasing number of complications. Multiple complications were inversely associated with age: patients with two or more complications were significantly younger than patients without any microvascular complications. In other regions, studies that have looked at age of onset of diabetes have found that early onset diabetes, usually defined as $<45$ years, increases disease severity. This observation suggests that patients with early onset of diabetes have a more aggressive disease and are prone to develop more complications at an earlier age [11-13]. Genetic determinants of diabetes are likely to be more common in patients with early onset of diabetes [14]. Our report agrees with other studies that the early onset of diabetes could be a helpful prognostic variable to predict the development of microvascular complications.

$\mathrm{HbAlc}$ was the only factor significantly associated with all three microvascular complications, although hypertension (current or under treatment) achieved borderline significance $(P=0.06)$. This finding implies that careful diabetic control is a worthwhile and achievable goal for minimizing the excess disability and costs associated with diabetes [15]. Of interest, each individual microcirculatory complication was associated with its own somewhat different pattern of risk factors. In addition to $\mathrm{HbAlc}$ levels and hypertension, these included patient age, family history, duration of diabetes, insulin use, and body mass index. In particular, concerning body mass index, it is well known that obesity predisposes to type 2 diabetes, a condition for which even a moderate weight loss can improve insulin resistance and chronic hyperglycemia, both of which are related to microvascular complications $[16,17]$.

This study has several weaknesses: it is cross-sectional and therefore not the best design for studying temporal changes. Although the possibility of interviewer bias exists, we believe that it should not affect our main findings since information about diabetic complications and treatment are not subjective and were corroborated with data retrieved from clinical charts. Also, participants were entirely voluntary and interviews were conducted by trained personnel. Since it is based on a clinic population, we have no information about the number of deaths or the causes of antecedent mortality, which could skew our results. As an example, smoking could be a major causative factor for deaths in this clinic population. Since this is a single institution study, the results and in particular the prevalence of complications may not be generalized; however, it was conducted in the largest tertiary care hospital in Doha, which is the most representative institution in the country. Furthermore, we report only microvascular and not macrovascular complications.

The study strengths are that the information comes from a carefully followed group of adult diabetics from a region with a high prevalence of diabetes. The Qatari and non-Qatari were similar, indicating that the findings are likely to be generalizable to the large population of male diabetics in the Middle East and perhaps to other regions. Another strength of the study is that it provides a road map to help guide diabetologists to compare and assess the likelihood of development of microvascular complications in their patients, as well as to determine the relative importance of individual risk factors for the individual type of complications.

In conclusion, this report provides information about the prevalence of individual and multiple microvascular complications among diabetics in Qatar, which has among the highest incidence rates of diabetes globally. The frequency of individual microvascular complications is generally similar to reports based on similarly aged patients from the same region [18-21] and from other regions [22-24]. However, unlike many previous reports which focus on a single complication, this report provides information for clinicians about the timing, the overlap, the pattern of development, and the risk factors associated with multiple microvascular complications. This information can be useful to diabetologists and clinicians responsible for the management of the large group of diabetics who develop microvascular complications. 


\section{Ethical Approval}

The Office of Research at Weill Cornell Medical CollegeQatar reviewed the proposal and determined that the study was exempt from Qatari and United States (US) human subjects' protection regulations and therefore did not require review by an institutional review board (IRB number 20120024). The Research Committee at Hamad Medical Corporation determined that the study was exempt, according to the rules and regulations for research at Hamad Medical Corporation and allowed access to their facilities (Research proposal number 12286/12).

\section{Conflicts of Interest}

The authors declare that there is no conflict of interest regarding the publication of this article.

\section{Authors' Contributions}

All authors have made substantive intellectual contributions to the study. Sohaila Cheema, Ravinder Mamtani, Albert B. Lowenfels, Mahmoud Zirie, Amin Jayyousi, Ziyad Mahfoud, Hekmat Alrouh, Amit Abraham, and Patrick Maisonneuve conceptualized and designed the study. Amit Abraham, Hekmat Alrouh, Sura Al-Samraye, Buthaina Ibrahim, and Ibrahim Mohammed Al-Janahi conducted the study and collected the data. Patrick Maisonneuve and Albert B. Lowenfels reviewed and analyzed the data. Sohaila Cheema, Patrick Maisonneuve, Albert B. Lowenfels, and Ravinder Mamtani prepared and formalized the manuscript. Amit Abraham, Hekmat Alrouh, Sura Al-Samraye, Mahmoud Zirie, Amin Jayyousi, Buthaina Ibrahim, Ziyad Mahfoud, and Ibrahim Mohammed Al-Janahi reviewed and edited the manuscript. All authors critically appraised, reviewed, and approved the final version of the manuscript submitted for publication.

\section{Acknowledgments}

The work was supported by the Institute for Population Health (previously Division of Global and Public Health), Weill Cornell Medicine-Qatar and Qatar Foundation, through the Weill Cornell Medicine-Qatar Biomedical Research Program.

\section{Supplementary Materials}

Supplementary data include characteristics of male and female participants and analysis of factors associated with microvascular complications, adjusted for age, sex, and duration of diabetes. (Supplementary Materials)

\section{References}

[1] L. Alhyas, A. McKay, and A. Majeed, "Prevalence of type 2 diabetes in the states of the Co-operation Council for the Arab States of the Gulf: a systematic review," PLoS One, vol. 7, no. 8, article e40948, 2012.

[2] A. Bener, E. J. Kim, F. Mutlu et al., "Burden of diabetes mellitus attributable to demographic levels in Qatar: an emerging public health problem," Diabetes \& Metabolic Syndrome: Clinical Research \& Reviews, vol. 8, no. 4, pp. 216-220, 2014.

[3] C. R. Pullinger, I. D. Goldfine, S. Tanyolaç et al., "Evidence that an HMGA1 gene variant associates with type 2 diabetes, body mass index, and high-density lipoprotein cholesterol in a Hispanic-American population," Metabolic Syndrome and Related Disorders, vol. 12, no. 1, pp. 25-30, 2014.

[4] K. H. K. Chan, Y. T. Huang, Q. Meng et al., "Shared molecular pathways and gene networks for cardiovascular disease and type 2 diabetes mellitus in women across diverse ethnicities," Circulation Cardiovascular Genetics, vol. 7, no. 6, pp. 911919, 2014.

[5] A. Zabetian, H. M. Keli, J. B. Echouffo-Tcheugui, K. M. V. Narayan, and M. K. Ali, "Diabetes in the Middle East and North Africa," Diabetes Research and Clinical Practice, vol. 101, no. 2, pp. 106-122, 2013.

[6] L. Litwak, S. Y. Goh, Z. Hussein, R. Malek, V. Prusty, and M. E. Khamseh, "Prevalence of diabetes complications in people with type 2 diabetes mellitus and its association with baseline characteristics in the multinational A1chieve study," Diabetology and Metabolic Syndrome, vol. 5, no. 1, p. 57, 2013.

[7] P. Romero-Aroca, I. Mendez-Marin, M. Baget-Bernaldiz, J. Fernandez-Ballart, and E. Santos-Blanco, "Review of the relationship between renal and retinal microangiopathy in diabetes mellitus patients," Current Diabetes Reviews, vol. 6, no. 2, pp. 88-101, 2010.

[8] A. Girach, D. Manner, and M. Porta, "Diabetic microvascular complications: can patients at risk be identified? A review," International Journal of Clinical Practice, vol. 60, no. 11, pp. 1471-1483, 2006.

[9] World Health Organization (WHO), "Global Adult Tobacco Survey: Qatar fact sheet 2013,” June 2018, http://www.emro. who.int/images/stories/tfi/documents/FACT_SHEETS/FS_ GATS_Qatar_2013.pdf?ua=1.

[10] C. Willi, P. Bodenmann, W. A. Ghali, P. D. Faris, and J. Cornuz, "Active smoking and the risk of type 2 diabetes: a systematic review and meta-analysis," Journal of the American Medical Association, vol. 298, no. 22, pp. 2654-2664, 2007.

[11] J. Wong, L. Molyneaux, M. Constantino, S. M. Twigg, and D. K. Yue, "Timing is everything: age of onset influences long-term retinopathy risk in type 2 diabetes, independent of traditional risk factors," Diabetes Care, vol. 31, no. 10, pp. 1985-1990, 2008.

[12] W. Zou, L. Ni, Q. Lu et al., "Diabetes onset at 31-45 years of age is associated with an increased risk of diabetic retinopathy in type 2 diabetes," Scientific Reports, vol. 6, no. 1, article 38113, 2016.

[13] T. A. Hillier and K. L. Pedula, "Complications in young adults with early-onset type 2 diabetes: losing the relative protection of youth," Diabetes Care, vol. 26, no. 11, pp. 2999-3005, 2003.

[14] S. H. Song and C. A. Hardisty, "Early onset type 2 diabetes mellitus: a harbinger for complications in later years-clinical observation from a secondary care cohort," QJM, vol. 102, no. 11, pp. 799-806, 2009.

[15] F. Ismail-Beigi, "Clinical practice. Glycemic management of type 2 diabetes mellitus," The New England Journal of Medicine, vol. 366, no. 14, pp. 1319-1327, 2012.

[16] M. Greco, E. Chiefari, T. Montalcini et al., "Early effects of a hypocaloric, mediterranean diet on laboratory parameters in obese individuals," Mediators of Inflammation, vol. 2014, Article ID 750860, 8 pages, 2014. 
[17] P. J. Joris, J. Plat, Y. H. A. M. Kusters et al., "Diet-induced weight loss improves not only cardiometabolic risk markers but also markers of vascular function: a randomized controlled trial in abdominally obese men," The American Journal of Clinical Nutrition, vol. 105, no. 1, pp. 23-31, 2017.

[18] K. Al-Rubeaan, A. M. Abu El-Asrar, A. M. Youssef et al., "Diabetic retinopathy and its risk factors in a society with a type 2 diabetes epidemic: a Saudi National Diabetes Registry-based study," Acta Ophthalmologica, vol. 93, no. 2, pp. e140-e147, 2015.

[19] M. Elshafei, H. Gamra, R. Khandekar, M. A. I. Hashimi, A. Pai, and M. F. Ahmed, "Prevalence and determinants of diabetic retinopathy among persons $\geq 40$ years of age with diabetes in Qatar: a community-based survey," European Journal of Ophthalmology, vol. 21, no. 1, pp. 39-47, 2011.

[20] F. Al-Mahroos and K. Al-Roomi, "Diabetic neuropathy, foot ulceration, peripheral vascular disease and potential risk factors among patients with diabetes in Bahrain: a nationwide primary care diabetes clinic-based study," Annals of Saudi Medicine, vol. 27, no. 1, pp. 25-31, 2007.

[21] S. Hajar, A. Al Hazmi, M. Wasli, A. Mousa, and M. Rabiu, "Prevalence and causes of blindness and diabetic retinopathy in southern Saudi Arabia," Saudi Medical Journal, vol. 36, no. 4, pp. 449-455, 2015.

[22] H. Yokoyama, M. Oishi, H. Takamura et al., "Large-scale survey of rates of achieving targets for blood glucose, blood pressure, and lipids and prevalence of complications in type 2 diabetes (JDDM 40)," BMJ Open Diabetes Research \& Care, vol. 4, no. 1, article e000294, 2016.

[23] T. H. Lee, H. J. Ryu, P. W. Chung, W. S. Lim, and M. Y. Chung, "The prevalence of diabetic complications in Korea," The Korean Journal of Internal Medicine, vol. 2, no. 1, pp. 42-48, 1987.

[24] A. D. Deshpande, M. Harris-Hayes, and M. Schootman, "Epidemiology of diabetes and diabetes-related complications," Physical Therapy, vol. 88, no. 11, pp. 1254-1264, 2008 . 


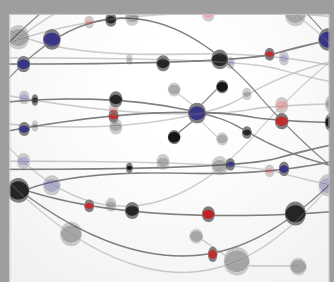

The Scientific World Journal
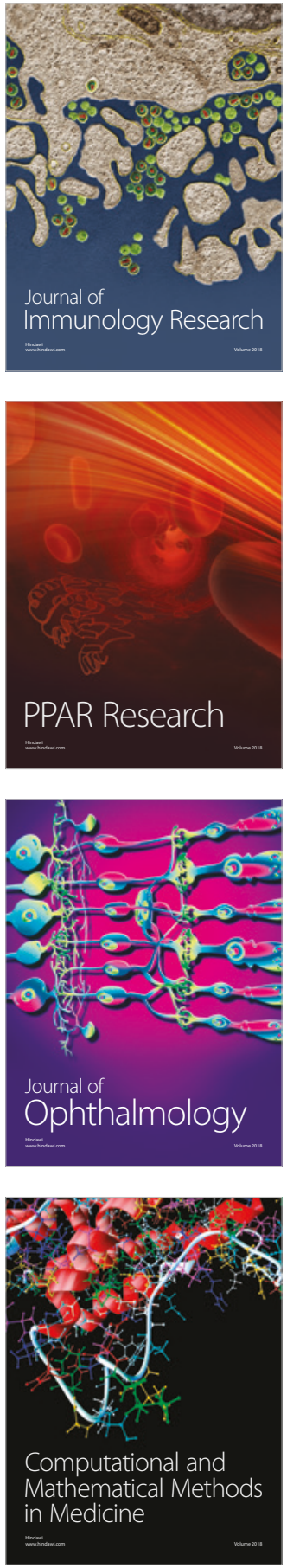

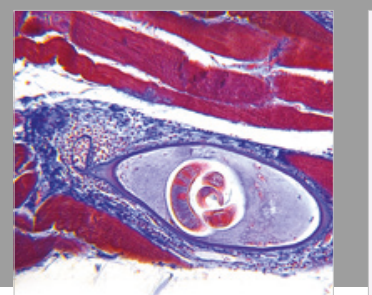

Gastroenterology Research and Practice

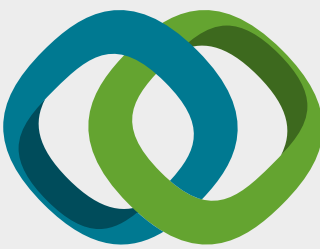

\section{Hindawi}

Submit your manuscripts at

www.hindawi.com
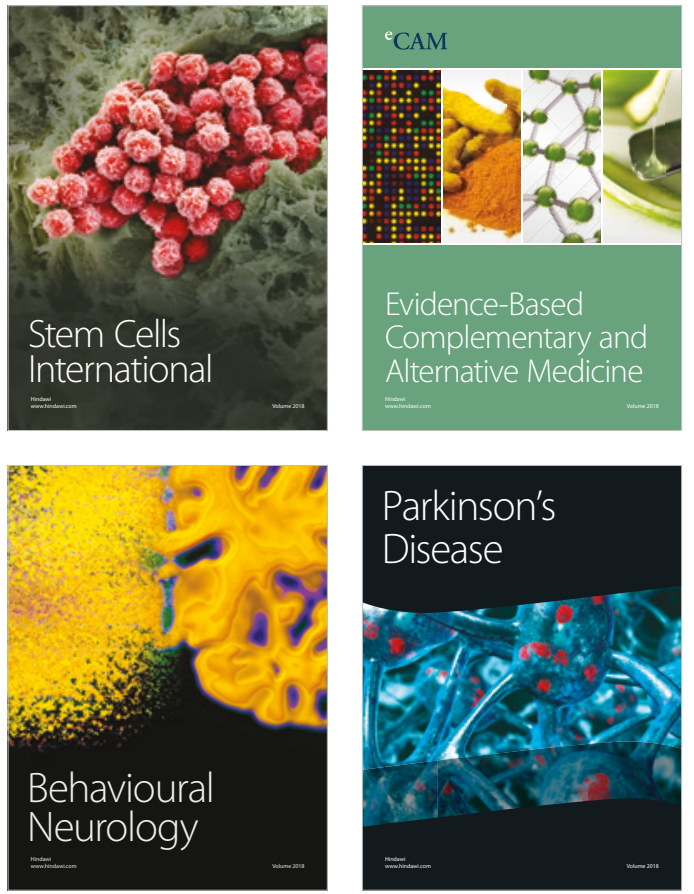

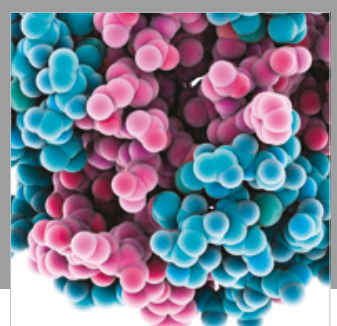

ournal of

Diabetes Research

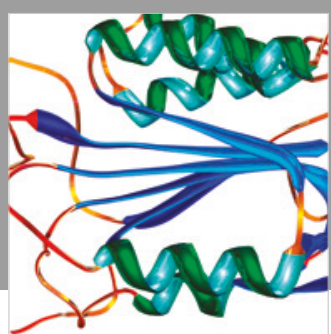

Disease Markers
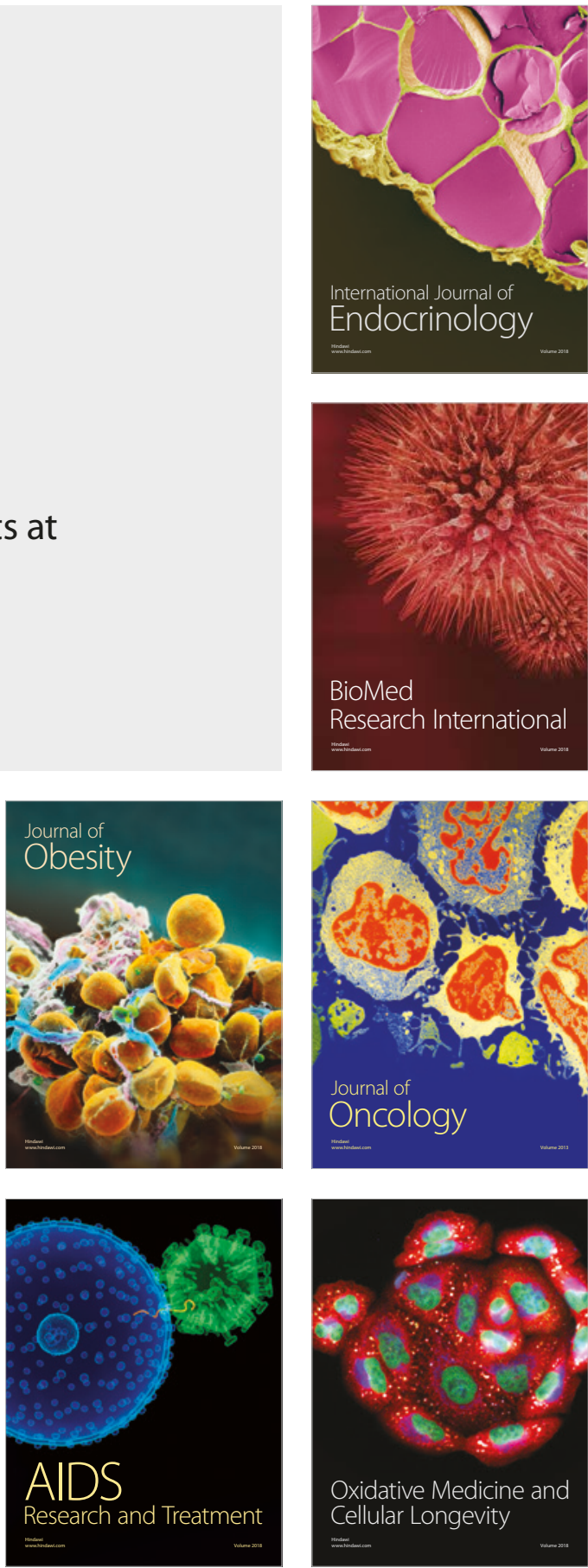